\title{
UNRAMIFIED DOUBLE COVERINGS OF HYPERELLIPTIC SURFACES. II
}

\author{
H. M. FARKAS \\ (Communicated by Irwin Kra)
}

\begin{abstract}
In this note we illustrate how to count the fixed points of a lift of the hyperelliptic involution to a smooth unramified double cover. In this way we obtain a new proof of the assertion that only $\left(\begin{array}{c}2 g+2 \\ 2\end{array}\right)$ of the double covers are hyperelliptic and classify the remaining covers in terms of $\tilde{g}$-hyperellipticity.
\end{abstract}

Introduction. In [F1] the author proved that among the $2^{2 g}-1$ unramified double coverings of a hyperelliptic surface $S$ of genus $g$ there are $\left(\begin{array}{c}2 g+2 \\ 2\end{array}\right)$ hyperelliptic surfaces while the remaining $2^{2 g}-1-\left(\begin{array}{c}2 g+2 \\ 2\end{array}\right)$ are not hyperelliptic. Recently, E. Bujalance $[\mathbf{B}]$ classified all the unramified double coverings of hyperelliptic surfaces using the representation of compact Riemann surfaces as quotient spaces of Fuchsian groups $[\mathbf{M}]$. The result is that all unramified double coverings are $p$ hyperelliptic for various $p$ between 0 and $[(g-1) / 2]$. In this note we shall show how to obtain Bujalance's result in a straightforward way by simply lifting the hyperelliptic involution from $S$ to the unramified double covering and counting its fixed points. In this note we only consider the case of "double coverings" and use a special method for this case which does not generalize. We do point out however that the method used here can be utilized to answer the question which unramified double covers of a $\tilde{g}$-hyperelliptic surface of genus $g$ remain $\tilde{g}$-hyperelliptic. The only additional ingredient is first determining to which double covers the $\tilde{g}$-hyperelliptic involution can be lifted. In another publication [F2] we consider the general problem of lifting the hyperelliptic involution to any finite unramified abelian covering.

1. It follows from general covering space theory that an unramified double covering of $S$ is determined by a subgroup, $K$, of index 2 of $\pi_{1}(S)$. Furthermore, if we denote by $h$ the natural homomorphism of $\pi_{1}(S)$ onto the quotient group of $\pi_{1}(S)$ modulo its commutator subgroup which we shall as usual denote by $\mathcal{H}^{1}(S)$, and let $\mathcal{K}=h(K)$, then it is immediate that $\alpha \in K$ if and only if $h(\alpha) \in \mathcal{K}$. This is obvious because $K$ contains the commutator subgroup of $\pi_{1}(S)$.

Let $S$ be a hyperelliptic surface of genus $g$ together with the canonical homology basis for $\not^{1}(S)$ pictured in Figure 1.

In Figure $1 S$ is represented as a branched two-sheeted cover of the sphere with branch points $P_{0}, P_{1}, \ldots, P_{2 g+1}$. The hyperelliptic involution $H$ on $S$ is the sheet interchange and the fixed points are the points $P_{i}, i=0, \ldots, 2 g+1$. Let $L_{i}$, $i=1, \ldots, 2 g+1$ be paths on $S$ joining $P_{0}$ to $P_{i}$ as indicated in Figure 1 .

Consider $\pi_{1}\left(S, P_{0}\right)$ and the curves $H\left(L_{i}\right) L_{i}^{-1}$ as elements of $\pi_{1}\left(S, P_{0}\right)$. Then $h\left[H\left(L_{i}\right) L_{i}^{-1}\right] \in \mathcal{H}^{1}(S)$ and computations made in [FK, pp. 301-306] show that the

Received by the editors October 3, 1985 and, in revised form, May 9, 1986.

1980 Mathematics Subject Classification (1985 Revision). Primary 30F 10; Secondary 14 H30. 


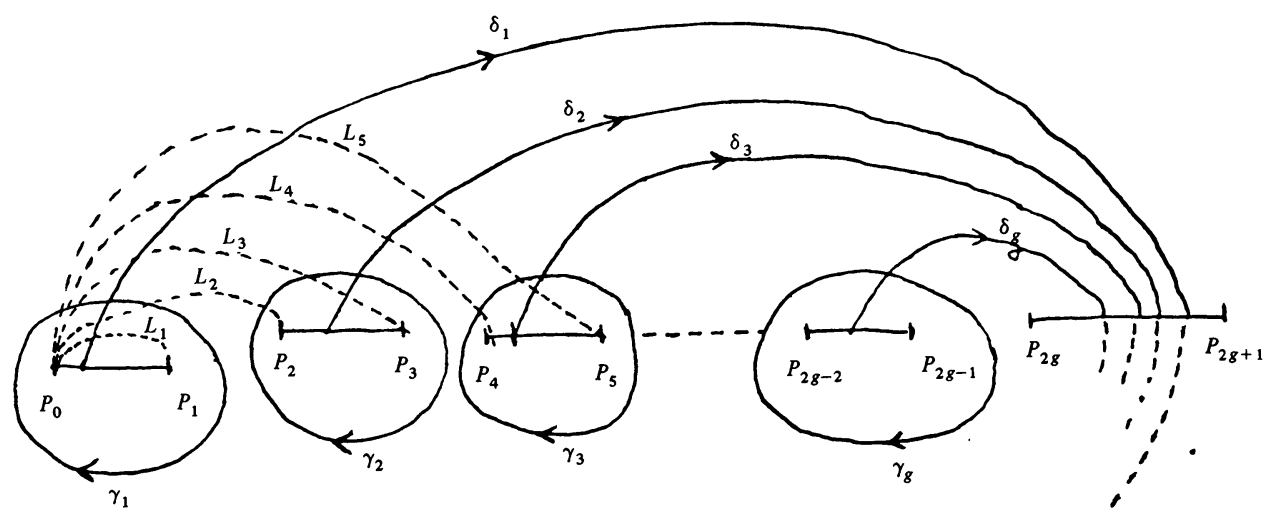

FIGURE 1

following matrix equation is satisfied:

$$
\left[\begin{array}{c}
h\left[H\left(L_{1}\right) L_{1}^{-1}\right. \\
\vdots \\
h\left[H\left(L_{2 g}\right) L_{2 g}^{-1}\right]
\end{array}\right]=A\left[\begin{array}{c}
\gamma_{1} \\
\vdots \\
\gamma_{g} \\
\delta_{1} \\
\vdots \\
\delta_{g}
\end{array}\right]
$$

where $A$ is the $2 g \times 2 g$ matrix

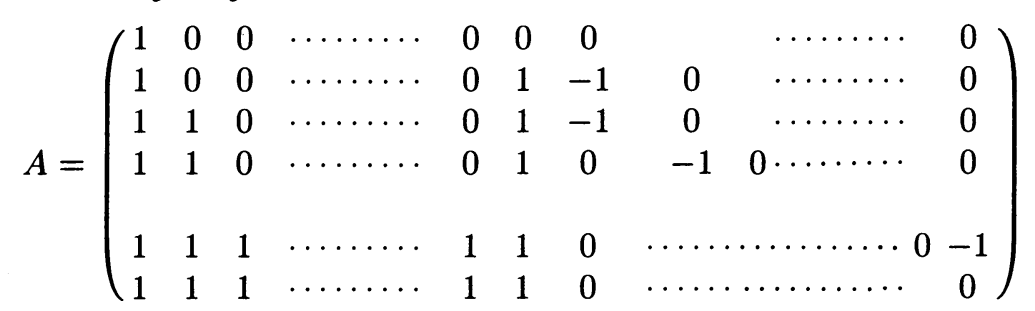

and $h\left[H\left(L_{2 g+1}\right) L_{2 g+1}^{-1}\right]=\delta_{1}$.

It is clear from the above representation that the intersection numbers $h\left[H\left(L_{i}\right) L_{i}^{-1}\right]$ and $h\left[H\left(L_{j}\right) L_{j}^{-1}\right]$ is always \pm 1 when $i \neq j$ and equals zero for $i=j$. In fact for $i<j$ the intersection number is +1 . Furthermore if we consider the $2 g$ row vectors of the matrix $A$ as elements of $(Z / 2)^{2 g}$ adjoin $(0,0 \cdots 0,1,0 \cdots 0)$, and denote this set by $W$, then these $2 g+1$ elements have the following property:

LEMMA 1. Every nonzero element of $(Z / 2)^{2 g}$ is uniquely expressible as a sum of $k$ elements of $W$ with $1 \leq k \leq g$.

ProOF. We first observe that $(Z / 2)^{2 g}$ has $2^{2 g}-1$ nonzero elements and that $\sum_{k=1}^{g}\left(\begin{array}{c}2 g+1 \\ k\end{array}\right)=2^{2 g}-1$. Hence to complete the proof we need only show that all the elements of $(Z / 2)^{2 g}$ generated this way are distinct. Since the rows of $A$ are linearly independent and $(0 \cdots 0,1,0 \cdots 0)$ is the sum of all the rows of $A,(0 \cdots 0,1,0 \cdots 0)$ lies in no proper subspace of the row space of $A$. Hence any collection of $2 g$ elements from $W$ is a linearly independent set. If two distinct sums were equal then we would have a linear relation between at most $2 g$ elements of $W$ which is impossible. 
2. Let $S$ be a hyperelliptic surface of genus $g$ and $\hat{S}$ be an unramified double covering of $S$. Let $H$ denote the hyperelliptic involution on $S$. Since $H$ acts as - Id on $H^{1}(S), H$ can be lifted to an involution $\hat{H}$ of $\hat{S}$ which fixes both points of $\hat{S}$ which lie over the point $P_{0} \in S$.

From general covering space theory we know that the points of $\hat{S}$ can be taken to be equivalence classes of ordered pairs $(P, J)$ where $P \in S$ and $J$ is a path joining $P_{0}$ to $P . \quad\left(P_{1}, J_{1}\right)$ is equivalent to $\left(P_{2}, J_{2}\right)$ provided $P_{1}=P_{2}$ and $J_{1} J_{2}^{-1} \in K$ where $K$ is the subgroup of $\pi_{1}\left(S, P_{0}\right)$ determining and determined by $\hat{S}$. We define $\hat{H}(P, J)=(H(P), H(J))$ and observe that it is well defined and a lift of $H$ which fixes the two points above $P_{0}$.

It is clear that the only possible other fixed points of $\hat{H}$ are the points lying above $P_{i}, i=1, \ldots, 2 g+1$. Note that if one point lying above $P_{i}$ is fixed so must the other one be. The points $\left(P_{i}, L_{i}\right), i=1, \ldots, 2 g+1$, are points on $\hat{S}$ lying over $P_{i} \in S$.

LEMMA 2. The two points above $P_{i}, i=1, \ldots, 2 g+1$, are fixed by $\hat{H}$ if and only if $h\left[H\left(L_{i}\right) L_{i}^{-1}\right] \in \mathcal{K}$.

ProOF. By our original observation at the beginning of $\S 1, H\left(L_{i}\right) L_{i}^{-1} \in K$ if and only if $h\left[H\left(L_{i}\right) L_{i}^{-1}\right] \in \mathcal{K}$. Therefore $\hat{H}\left(P_{i}, L_{i}\right)=\left(P_{i}, L_{i}\right)$ if and only if $H\left(L_{i}\right) L_{i}^{-1} \in K$ which is so if and only if $h\left[H\left(L_{i}\right) L_{i}^{-1}\right] \in \mathcal{K}$.

It thus follows that the number of fixed points of $\hat{H}$ on $\hat{S}$ is $2+2 l$ where $l$ is the number of indices $i, i=1, \ldots, 2 g+1$, for which $h\left[H\left(L_{i}\right) L_{i}^{-1}\right] \in \mathcal{K}$. Furthermore, in addition to $\hat{H}$ there is another lift of $H, \hat{H}_{1}$, where $\hat{H}_{1}=T \hat{H}$ with $T$ the fixed point free involution on $\hat{S}$ and the number of fixed points of $\hat{H}_{1}$ is $4 g+2-2 l$.

THEOREM. Let $\hat{S}$ be an unramified double cover of the surface $S$ of genus $g$. Then $\hat{S}$ is $\tilde{g}$-hyperelliptic for some $\tilde{g}, 0 \leq \tilde{g} \leq[(g-1) / 2]$. If $g$ is even there are $\left(\begin{array}{c}2 g+2 \\ 2 \tilde{g}+2\end{array}\right)$ different $\tilde{g}$-hyperelliptic surfaces $\hat{S}$ while if $g$ is odd there are for $0 \leq \tilde{g} \leq$ $((g-3) / 2)\left(\begin{array}{c}2 g+2 \\ 2 \tilde{g}+2\end{array}\right)$ different $\tilde{g}$-hyperelliptic surfaces and $\frac{1}{2}\left(\begin{array}{c}2 g+2 \\ g+1\end{array}\right)$ different $\tilde{g}$-hyperelliptic surfaces for $\tilde{g}=(g-1) / 2$. Finally each $\tilde{g}$ occurs with the given multiplicity, i.e. each $\tilde{g}$ occurs precisely that many times.

ProOF. Since $\hat{S}$ is an unramified double cover of $S$ there is a homomorphism $j: \mathcal{H}^{1}(S) \rightarrow Z / 2$ with kernel $\mathcal{K}$ and $\mathcal{K}$ determines $\hat{S}$. The homomorphism $j$ is determined by its action on the generators of $\mathcal{H}^{1}(S)$ so that each nontrivial homomorphism of $j$ onto $Z / 2$ is defined by a nonzero element in $(Z / 2)^{2 g}$.

There are clearly $2^{2 g}-1$ different nontrivial homomorphisms $j$ and associated with $j$ we have $\left(j\left(\delta_{1}\right), \ldots, j\left(\delta_{g}\right), j\left(\gamma_{1}\right), \ldots, j\left(\gamma_{g}\right)\right) \in(Z / 2)^{2 g}$. By Lemma 1 we have $\left(j\left(\delta_{1}\right), \ldots, j\left(\delta_{g}\right), j\left(\gamma_{1}\right), \ldots, j\left(\gamma_{g}\right)\right)$ is uniquely representable as a sum of $k$ elements of $W$ with $k \leq g$. Write $\left(j\left(\delta_{1}\right), \ldots, j\left(\delta_{g}\right), j\left(\gamma_{1}\right), \ldots, j\left(\gamma_{g}\right)\right)=\sum_{i=1}^{k} r_{i}$ where $r_{i} \in W$.

By definition an element $\gamma \in \mathcal{H}^{1}(S), \gamma=\sum a_{i} \gamma_{i}+b_{i} \delta_{i}$ is in $\mathcal{K}$ provided $\sum a_{i} j\left(\gamma_{i}\right)+b_{i} j\left(\delta_{i}\right) \underset{(2)}{=} 0$. If we denote $\gamma$ by $\left(\dot{a_{1}}, \ldots, a_{g}, b_{1}, \ldots, b_{g}\right)$ and $\left(j\left(\delta_{1}\right), \ldots\right.$, $\left.j\left(\delta_{g}\right), j\left(\gamma_{1}\right), \ldots, j\left(\gamma_{g}\right)\right)$ by $\left(\varepsilon_{1}^{\prime}, \ldots, \varepsilon_{g}^{\prime}, \varepsilon_{1}, \ldots, \varepsilon_{g}\right)$ then viewing $\left(\varepsilon_{1}^{\prime}, \ldots, \varepsilon_{g}^{\prime}, \varepsilon_{1}, \ldots, \varepsilon_{g}\right)$ as representing the element $\delta=\sum \varepsilon_{i}^{\prime} \gamma_{i}+\varepsilon_{i} \delta_{i}$ in $\mathcal{H}^{1}(S)$ the condition that $\gamma \in \mathcal{K}$ can be written as the intersection number of $\gamma$ and $\delta$ is $0 \bmod 2$. As before writing 
$\delta=\sum_{i=1}^{k} r_{i}$ with $k \leq g$ the intersection number of $\gamma$ and $\delta$ is the sum of the intersection numbers of $\gamma$ and the $r_{i}$.

Recall however that for each $i, h\left[H\left(L_{i}\right) L_{i}^{-1}\right] \in W$ and therefore that $h\left[H\left(L_{i}\right) L_{i}^{-1}\right]$ has intersection number \pm 1 with each $r_{i}$. It then follows that for $k$ odd $k=2 n-1, n=1, \ldots,[(g+1) / 2]$ there are precisely $2 n-1$ indices $i$ for which $H\left(L_{i}\right) L_{i}^{-1} \in K$ so that $\hat{H}$ has precisely $4 n$ fixed points. Therefore $\hat{H}_{1}$ has precisely $4 g+4-4 n$ fixed points. Furthermore for $k$ even $k=2 n, n=1, \ldots,[g / 2]$, there are precisely $2 g+1-2 n$ indices $i$ for which $H\left(L_{i}\right) L_{i}^{-1} \in K$ so that $\hat{H}$ has precisely $4 g+4-4 n$ fixed points.

If a compact Riemann surface of genus $2 g-1$ has an involution with $4 g+4-4 n$ fixed points it follows from the Riemann Hurwitz formula that the surface is $\tilde{g}$ hyperelliptic with $\tilde{g}=n-1$.

Each different choice of $k$ gives rise to a cover $\hat{S}$ and what we have seen is that $k=2 n-1$ and $k=2 n$ both give rise to $\tilde{g}$-hyperelliptic surfaces with $\tilde{g}=n-1$. The number of different covers we obtain with $k=2 n-1$ (resp. $k=2 n)$ is $\left(\begin{array}{c}2 g+1 \\ 2 n-1\end{array}\right)$ (resp. $\left.\left(\begin{array}{c}2 g+1 \\ 2 n\end{array}\right)\right)$. Hence the number of $(n-1)$-hyperelliptic surfaces is

$$
\left(\begin{array}{c}
2 g+1 \\
2 n-1
\end{array}\right)+\left(\begin{array}{c}
2 g+1 \\
2 n
\end{array}\right)=\left(\begin{array}{c}
2 g+2 \\
2 n
\end{array}\right)=\left(\begin{array}{c}
2 g+2 \\
2 \tilde{g}+2
\end{array}\right) .
$$

The above remarks are correct provided $n \leq g / 2$ and for $g$ even we therefore have proved the theorem. If $g$ is odd then when $k=g, k=2 n-1$ with $n=$ $(g+1) / 2$ and the number of different ways of obtaining covers with $k=g$ is as we observed before

$$
\left(\begin{array}{c}
2 g+1 \\
2 n-1
\end{array}\right)=\left(\begin{array}{c}
2 g+1 \\
g
\end{array}\right)=\left(\begin{array}{c}
2 g+1 \\
g+1
\end{array}\right)
$$

This can therefore be written as $\frac{1}{2}\left(\begin{array}{c}2 g+2 \\ g+1\end{array}\right)$ which equals $\frac{1}{2}\left(\begin{array}{c}2 g+2 \\ 2 n\end{array}\right)$ which equals $\frac{1}{2}\left(\begin{array}{c}2 g+2 \\ 2 \tilde{g}+2\end{array}\right)$.

3. In this note we have used the set $W$ to generate the elements of $(Z / 2)^{2 g}$ and this was the key to proving the theorem. The subset $W$ is quite classical and has been important in the theory of theta functions. This, as far as the author is aware, is the first application to other areas. Furthermore, the same ideas can be used to answer the question as to which unramified double covers of a $\tilde{g}$-hyperelliptic surface of genus $g$ remain $\tilde{g}$-hyperelliptic and a classification can be obtained. The only additional complication is first determining to which covers the $\tilde{g}$-hyperelliptic involution can be lifted. Hence this note was written not so much to prove the theorem as to exhibit the method. The theorem is as already mentioned due to Bujalance [B].

The ideas used here do not easily generalize to unramified abelian covers of degree greater than two. The theorem however does not generalize and this has been treated in [F2].

\section{REFERENCES}

[B] E. Bujalance, A classification of unramified double coverings of hyperelliptic Riemann surfaces (preprint), Arch. Math. (Basel) (to appear).

[F1] H. M. Farkas, Unramified double coverings of hyperelliptic surfaces, J. Analyse Math. 30 (1976), 150-155. 
[F2] _ Unramified coverings of hyperelliptic Riemann surfaces (Preprint).

[FK] H. M. Farkas and I. Kra, Riemann surfaces, Springer-Verlag, Berlin and New York, 1980.

[M] C. Maclachlan, Smooth coverings of hyperelliptic surfaces, Quart. J. Math. Oxford Ser. (2) 22 (1971), 117-123.

Institute of Mathematics, The Hebrew University, Jerusalem, IsRael 